\title{
CORRELAÇÃO RADIOLÓGICA E HISTOLÓGICA DE LESÕES MAMÁRIAS NÃO-PALPÁVEIS EM PACIENTES SUBMETIDAS A MARCAC̣ÃO PRÉ-CIRÚRGICA, UTILIZANDO-SE O SISTEMA BI-RADS*
}

\author{
Vaneska de Carvalho Melhado ${ }^{1}$, Beatriz Regina Alvares ${ }^{2}$, Orlando José de Almeida ${ }^{3}$
}

Resumo OBJETIVO: Avaliar as categorias 3, 4 e 5 da classificação BI-RADS (Breast Imaging Reporting and Data System) como fator preditivo para malignidade, correlacionando os achados mamográficos e histológicos em lesões não-palpáveis da mama. MATERIAIS E MÉTODOS: Estudo analítico descritivo de 169 mulheres submetidas a biópsia cirúrgica, após localização estereotáxica de lesões mamárias não-palpáveis. As mamografias dessas pacientes foram classificadas de acordo com a quarta edição do BI-RADS, avaliando-se as categorias 3, 4 (A, B e C) e 5. Correlacionaram-se os achados mamográficos com os exames histológicos das lesões, avaliando-se o valor preditivo positivo em cada categoria. RESULTADOS: No total de 169 casos, foram diagnosticados 42 casos de câncer (24,8\%). Destes, houve apenas um caso na categoria 3, 19 casos na categoria 4 e 22 casos na categoria 5 . Os valores preditivos positivos para as categorias 3, 4A, 4B, 4C e 5 foram, respectivamente, de 3,4\%, 10,3\%, 11,3\%, 36\% e 91,7\%. As microcalcificações foram o achado mais freqüente relacionado à doença maligna, ocorrendo em $61,5 \%$ do total. CONCLUSÃO: Este estudo demonstrou que a classificação BI-RADS permite predizer com segurança que há alta suspeição de malignidade para achados classificados na categoria 5 e diminuta chance para os achados da categoria 3 . Quanto à categoria 4, foi constatada elevação progressiva dos valores preditivos positivos nas subcategorias $A, B$ e C, mostrando que esta subdivisão contribui de forma mais detalhada e precisa na indicação de lesões suspeitas para malignidade.

Unitermos: Câncer de mama; Mamografia; BI-RADS; Diagnóstico histológico.

Abstract Radiological and histological correlation of non-palpable breast lesions in patients submitted to preoperative marking according to BI-RADS classification.

OBJECTIVE: To evaluate the positive predictive value for BI-RADS (Breast Imaging Reporting and Data System) categories 3,4 and 5, correlating mammographic and histological diagnosis in non-palpable breast lesions. MATERIALS AND METHODS: Analytical-descriptive study of 169 women submitted to stereotactic localization for surgical biopsy of non-palpable breast lesions. Mammographic and histological findings were correlated, analyzing the predictive positive value for each category. RESULTS: Forty-two $(24.8 \%)$ cases were diagnosed with breast cancer - only one in category 3, 19 in category 4, and 22 in category 5 . The positive predictive value for categories $3,4 \mathrm{~A}, 4 \mathrm{~B}, 4 \mathrm{C}$ and 5 were, respectively, $3.4 \%, 10.3 \%, 11.3 \%, 36 \%$ and $91.7 \%$. Microcalcifications were the most frequent finding related to malignancy, present in $61.5 \%$ of these cases. CONCLUSION: The present study has demonstrated that BI-RADS allows a safe prediction of high suspicion of malignancy in lesions category 5 and low suspicion for category 3 . As regards the category 4, the positive predictive value has shown a progressive increase in subcategories A, B and C, demonstrating that this subclassification represents an invaluable contribution for a more detailed and accurate assessment of lesions suspicious for malignancy.

Keywords: Breast cancer; Mammography; BI-RADS; Histological diagnosis.

* Trabalho realizado no Setor de Radiologia do Centro de Atenção Integral à Saúde da Mulher (CAISM) da Universidade Estadual de Campinas, Campinas, SP. Estudo financiado pela Fundação de Amparo à Pesquisa do Estado de São Paulo (Fapesp)

1. Médica Residente na área de Ginecologia e Obstetrícia da Faculdade de Ciências Médicas da Universidade Estadual de Campinas.

2. Professora Assistente do Departamento de Radiologia da Faculdade de Ciências Médicas da Universidade Estadual de Campinas.

3. Responsável pelo Senviço de Mamografia - Policlínica II/ Prefeitura Municipal de Campinas.

Endereço para correspondência: Dra. Vaneska de Carvalho Melhado. Rua 3-A, 94, Vila Alemã. Rio Claro, SP, 13506-660. E-mail: vaneskacm@hotmail.com

Recebido para publicação em 7/10/2005. Aceito, após revisão, em 6/4/2006.

\section{INTRODUÇÃO}

A mamografia é o método mais específico e sensível para o diagnóstico do câncer de mama em sua forma mais precoce ${ }^{(1)}$. $\mathrm{O}$ rastreamento mamográfico anual em mulheres acima de 40 anos identifica 100 a 200 casos novos de lesões suspeitas em cada 20.000 mamografias, que se apresentam como lesões não-palpáveis e que necessitam de estudo histológico, sendo uma das opções a sua localização pré-operatória $^{(2)}$. Assim, apesar do bom desempenho da mamografia na identificação da neoplasia mamária em estágio inicial, apenas 15\% a 30\% das lesões não-palpáveis submetidas à biopsia cirúrgica são malignas ${ }^{(3)}$.

Isso provocou a elaboração de propostas para classificar os achados mamográficos, visando melhorar o desempenho do método e reduzir a frequiência de biópsias com diagnóstico benigno ${ }^{(4)}$.

O Colégio Americano de Radiologia (ACR - American College of Radiology) desenvolveu a classificação Breast Imaging Reporting and Data System (BI-RADS) 
para padronizar a terminologia do relatório mamográfico, os achados radiológicos e as recomendações a serem tomadas. $\mathrm{Na}$ quarta edição do BI-RADS, de novembro de 2003, foram propostas sete categorias para os achados mamográficos: negativos para malignidade (1), benignos (2), provavelmente benignos (3), suspeitos para malignidade (4), altamente suspeitos para malignidade (5), com malignidade comprovada (6) e que necessitam de avaliação adicional (0). A categoria 4 foi subdividida em $\mathrm{A}, \mathrm{B}$ e $\mathrm{C}^{(\mathbf{5 , 6})}$.

Este estudo teve como objetivos avaliar o sistema BI-RADS (quarta edição) como fator preditivo de suspeição para malignidade em lesões não-palpáveis da mama correlacionando os achados radiológicos e histológicos, e verificar quais os achados mais relevantes para o câncer de mama em cada categoria (3, 4A, 4B, 4C e 5).

\section{MATERIAIS E MÉTODOS}

Realizou-se a análise mamográfica de 169 lesões não-palpáveis de pacientes biopsiadas no período de setembro de 2003 a abril de 2004. Esta avaliação foi feita por médicos radiologistas com experiência em diagnóstico mamográfico, sendo os achados classificados de acordo com o sistema BI-RADS e avaliadas as categorias 3, 4A, $4 \mathrm{~B}, 4 \mathrm{C}$ e 5 .

As mamografias avaliadas no estudo foram realizadas em mamógrafo Mammomat 3000 Nova (Siemens), nas incidências crânio-caudal e médio-lateral oblíqua, além de incidências complementares como magnificação e compressão focal. As pacientes foram submetidas à marcação pré-cirúrgica através de estereotaxia por mamografia em $90,53 \%$ (153) dos casos, ou por ultra-sonografia em $9,46 \%$ (16).

O tecido mamário obtido nas biópsias cirúrgicas foi processado para cortes em blocos de parafina e coloração pela hematoxilina-eosina e os diagnósticos foram elaborados por médicos patologistas com experiência em patologia mamária.

O estudo foi do tipo analítico descritivo, para avaliar a concordância entre a classificação BI-RADS atualizada e os diagnósticos histológicos em lesões mamárias nãopalpáveis, através do cálculo do valor preditivo positivo (VPP). Procurou-se também correlacionar quais os achados radiológicos mais importantes relacionados à neoplasia maligna em cada categoria.

\section{RESULTADOS}

Entre as pacientes incluídas no estudo, $36,1 \%$ tinham menos de 50 anos, 36,7\% tinham entre 50 e 59 anos e 27,2\%, 60 anos ou mais de idade.

Do total de 169 casos analisados, a distribuição percentual dos diagnósticos mamográficos entre as categorias do BIRADS foi de $17,2 \%$ (29) para a categoria $3,68,6 \%$ (116) para a categoria 4 e 14,2\% (24) para a categoria 5. Avaliando somente a categoria 4, as suas subcategorias tiveram distribuição percentual de 25\% (29/116) para a subcategoria A, 53\% (62/116) para a subcategoria B e $22 \%(25 / 116)$ para a subcategoria C.

Houve 42 casos $(24,8 \%)$ de câncer mamário, sendo um na categoria 3 , três na categoria $4 \mathrm{~A}$, sete na categoria $4 \mathrm{~B}$, nove na categoria 4C e 22 na categoria 5. Os VPP foram, portanto, de 3,4\% (1/29) para BIRADS 3, 10,3\% (3/29) para BI-RADS 4A, $11,3 \%$ (7/62) para BI-RADS 4B, 36\% (9/ 25) para BI-RADS 4C e 91,7\% (22/24) para BI-RADS 5 (Tabela 1).

Entre as lesões classificadas como BIRADS 3, houve apenas um caso de câncer mamário, cujo aspecto radiológico foi de assimetria focal. Nas demais categorias, os achados mamográficos mais relacionados ao câncer mamário foram: para a categoria 4A, microcalcificações puntiformes com distribuição segmentar, em 66,7\% (2/3) dos casos; para a categoria 4B, microcalcificações puntiformes, amorfas e heterogêneas, em $57,1 \%$ (4/7) dos casos; para a categoria $4 \mathrm{C}$, distorção arquitetural com espículas, em 66,7\% (6/9) dos casos; para as le-

Tabela 1 Correlação do BI-RADS nas categorias $3,4 \mathrm{~A}, 4 \mathrm{~B}, 4 \mathrm{C}$ e 5 com os diagnósticos histológicos.

\begin{tabular}{|c|c|c|c|}
\hline \multirow[b]{2}{*}{ BI-RADS } & \multicolumn{3}{|c|}{ Diagnóstico histológico } \\
\hline & $\begin{array}{l}\text { Benigno } \\
\% \quad(\mathrm{n})\end{array}$ & $\begin{array}{l}\text { Maligno } \\
\% \quad(n)\end{array}$ & $\begin{array}{c}\text { Total } \\
\text { n (169) }\end{array}$ \\
\hline 3 & $96,6(28)$ & 3,4 (1) & 29 \\
\hline $4 \mathrm{~A}$ & $89,7(26)$ & $10,3(3)$ & 29 \\
\hline 4B & $88,7(55)$ & $11,3(7)$ & 62 \\
\hline $4 \mathrm{C}$ & $64,0(16)$ & $36,0(9)$ & 25 \\
\hline 5 & 8,3 (2) & 91,7 (22) & 24 \\
\hline
\end{tabular}

sões classificadas como BI-RADS 5, microcalcificações pleomórficas e ramificadas estavam presentes em 72,7\% (16/22) dos casos de câncer. No geral, os achados radiológicos mais relacionados à doença maligna foram as microcalcificações, presentes em $61,5 \%$ do total (Tabela 2).

A neoplasia mamária maligna mais freqüente foi o carcinoma ductal in situ em $59,5 \%$ (25/42), seguido por carcinoma ductal invasivo em 33,3\% (14/42), carcinoma lobular in situ em 4,8\% (2/42) e carcinoma lobular invasivo em um caso.

O diagnóstico de hiperplasia ductal atípica ocorreu em 7,1\% (12/169) do total de casos, todos eles na categoria 4 . Em 66,7\% $(8 / 12)$ do total, os achados foram as microcalcificações, e em 33,3\% (4/12), a distorção arquitetural. Os dois casos BI-RADS 4A apresentaram-se como grupamentos de microcalcificações redondas, lineares e puntiformes, tendendo a coalescer. Já a categoria 4B teve quatro casos de microcalcificações puntiformes, amorfas e heterogêneas, e um caso de grupamento de microcalcificações com distribuição linear. Na categoria $4 \mathrm{C}$, foram um caso de grupamento de microcalcificações pleomórficas com distribuição linear e quatro casos de distorção arquitetural.

\section{DISCUSSÃO}

A classificação BI-RADS representou a primeira tentativa de padronização dos achados mamográficos em termos descritivos, constituindo-se num importante instrumento para auxiliar tanto na suspeita de malignidade quanto na conduta a ser toma$\mathrm{da}^{(\mathbf{5 , 7 - 9})}$. Estudos que correlacionaram os achados mamográficos e histológicos em lesões mamárias não-palpáveis, utilizando a classificação BI-RADS, encontraram VPP para câncer de mama entre $12,3 \%$ e $47,8 \%{ }^{(\mathbf{4 , 7 , 8 , 1 0 - 1 2 )}}$. No presente estudo, $24,8 \%$ do total das lesões mamárias não-palpáveis biopsiadas tiveram o diagnóstico histológico de doença maligna.

O percentual de achados mamográficos provavelmente benignos submetidos à biópsia foi elevado $(17,2 \%)$, acima dos valores de outros estudos, que variaram entre $2 \%$ e $11 \%{ }^{(\mathbf{4 , 7 , 8 , 1 0 )}}$. Como $96,5 \%$ das lesões classificadas na categoria 3 foram benignas, isto contribuiu para a redução do 
Tabela 2 Distribuição dos achados radiológicos nas categorias BI-RADS 3, 4A, 4B, 4C e 5 em mulheres com neoplasia maligna da mama.

\begin{tabular}{|l|c|c|c|c|c|c|}
\hline \multirow{2}{*}{ Achados radiológicos } & \multicolumn{5}{|c|}{ BI-RADS } & \multicolumn{2}{c|}{ Total } \\
\cline { 2 - 7 } & 3 & $4 \mathrm{~A}$ & $4 \mathrm{~B}$ & $4 \mathrm{C}$ & 5 & $\mathrm{n}(\%)$ \\
\hline Assimetria focal & 1 & - & - & - & - & $1(2)$ \\
Assimetria focal com microcalcificações & - & - & - & 1 & - & $1(2)$ \\
Distorção arquitetural & - & - & - & 6 & - & $6(14)$ \\
Nódulo & - & - & 1 & 1 & 6 & $8(19)$ \\
Microcalcificações & - & 3 & 6 & 1 & 16 & $26(62)$ \\
\hline
\end{tabular}

VPP na avaliação global das categorias deste estudo. Ao analisarmos o VPP exclusivo da categoria 3 , o valor foi de $3,4 \%$, dentro da média de outros artigos ${ }^{(4,7,8,11)}$. O número elevado de biópsias cirúrgicas em lesões provavelmente benignas pode ser justificado pela dificuldade de acompanhamento mamográfico semestral em muitas pacientes oriundas de outros municípios e estados.

Ao correlacionar os achados radiológicos e histológicos nas lesões mamárias da categoria 4, o VPP em nosso estudo foi de $16,4 \%$, enquanto outros autores encontraram VPP entre $4 \%$ e $45 \% \%^{(4,7,8,10-12)}$.

No presente estudo, houve 12 casos de lesões mamárias classificadas como BIRADS 4 com o diagnóstico de hiperplasia ductal atípica que não foram incluídas no cálculo do VPP. Segundo Heywang-Köbrunner et al., a "hiperplasia ductal atípica representa carcinoma ductal in situ incompleto ou lesão borderline, cujo risco de malignização em comparação com a população normal encontra-se aumentado em quatro a cinco vezes" ${ }^{\prime(13)}$. Se os casos de hiperplasia ductal atípica, considerada uma lesão de risco, fossem incluídos no cálculo do VPP da categoria 4, observaríamos uma elevação para $26,7 \%$.

Ao analisar os achados nas subcategorias 4A, 4B e 4C encontramos, respectivamente, VPP de $10,3 \%, 11,3 \%$ e $36 \%$. Ao incluirmos as hiperplasias ductais atípicas nesta categoria, os valores mudariam para
$17,2 \%, 19,4 \%$ e $56 \%$. Estes achados demonstraram, portanto, uma sensibilidade crescente da classificação BI-RADS 4A, 4B e 4C para detectar as lesões suspeitas de câncer mamário e aquelas consideradas de risco para malignidade.

Não foram encontrados, na literatura, trabalhos mais recentes sobre os VPP das subcategorias 4A, 4B e 4C, não sendo possível, portanto, estabelecer correlação com os valores obtidos neste estudo ${ }^{(\mathbf{9 - 1 2})}$.

Em relação à categoria 5 , foi constatado VPP de 91,7\% para malignidade, estando de acordo com dados da literatura ${ }^{(4,7,8,10,11)}$. Nas lesões classificadas como BI-RADS 5, observaram-se um caso de adenose esclerosante e outro de cicatriz radiada, situações nas quais não é possível fazer o diagnóstico diferencial através da mamografia, sendo uma indicação obrigatória de biópsia excisional ${ }^{(6,14)}$.

\section{CONCLUSÃO}

O presente estudo demonstrou que a classificação BI-RADS permite predizer com segurança que existe alta suspeição para malignidade em lesões classificadas na categoria 5 e diminuta suspeição naquelas classificados como categoria 3. Quanto à categoria 4 , foi constatada elevação progressiva dos VPP nas subcategorias A, B e $C$, mostrando que esta subdivisão contribui de forma mais detalhada e precisa na indicação de lesões suspeitas para malignidade.

\section{REFERÊNCIAS}

1. Bellantone R, Rossi S, Lombardi CP, et al. Nonpalpable lesions of the breast. Diagnostic and therapeutic considerations. Minerva Chir 1994; 49:327-333.

2. Frasson A, Farante G, Sacchini V, et al. Localização pré-operatória de lesões mamárias não-palpáveis. Rev Bras Ginecol Obstet 1993;3:35-44.

3. Hall FM, Storella JM, Silverstone DM, Wyshak G. Nonpalpable breast lesions: recommendations for biopsy based on suspicion of carcinoma at mammography. Radiology 1988;167:353-358.

4. Orel SG, Kay N, Reynolds C, Sullivan DC. BIRADS categorization as a predictor of malignancy. Radiology 1999;211:845-850.

5. D’Orsi CJ. Illustrated Breast Imaging Reporting and Data System. 4th ed. Reston: American College of Radiology, 2003.

6. Rocha DC, Bauab SP. Atlas de imagem da mama. Correlação mamografia/ultra-sonografia, incluindo ressonância magnética e BI-RADS. $2^{\underline{a}}$ ed. Rio de Janeiro: Revinter, 2004.

7. Liberman L, Abramson AF, Squires FB, Glassman JR, Morris EA, Dershaw DD.The Breast Imaging Reporting and Data System: positive predictive value of mammographic features and final assessment categories. AJR Am J Roentgenol 1998;171: 35-40.

8. Bérubé M, Curpen B, Ugolini P, et al. Level of suspicion of mammographic lesion: use of features defined by BI-RADS lexicon and correlation with large-core breast biopsy. Can Assoc Radiol J 1998;49:223-228.

9. Vieira AV, Toigo FT. Classificação BI-RADS: categorização de 4.968 mamografias. Radiol Bras 2002;35:205-208.

10. Kestelman FP, Canella EO, Arvellos NA, et al. Classificação radiológica nas lesões não-palpáveis da mama. Análise de resultados do Hospital do Câncer III - INCA-MS. Radiol Bras 2001;34 (Supl 1):20.

11. Giannotti IA, Giannotti Filho O, Scalzaretto AP, Visentainer M, Elias S. Correlação entre diagnóstico por imagem e histologia de lesões não-palpáveis de mama. Rev Bras Cancer 2003;49:87-90.

12. Roveda Jr D, Campos MSDA, Ferezin PC, Ferreira ACP, Valle MJR. Valor preditivo das anormalidades mamográficas pelo sistema BI-RADS na ausência de lesões palpáveis das mamas. Radiol Bras 2001;34(Supl 1):21.

13. Heywang-Köbrunner SH, Schreer I, Dershaw DD, et al. Mama: diagnóstico por imagem. Correlação entre mamografia, ultra-sonografia, ressonância magnética, tomografia computadorizada e procedimentos intervencionistas. $1^{\underline{a}}$ ed. Rio de Janeiro: Revinter, 1999;141-155.

14. Stefenon CC, Carvalho AA, Djahjah MCR, Koch HA. Cicatriz radial/lesão esclerosante complexa: aspectos radiológicos com correlação clínica, ultra-sonográfica e anatomopatológica. Radiol Bras 2003;36:95-103. 